Dedicated to the 90th birthday anniversary of Professor G. Chogoshvili

\title{
GENERALIZED HEISENBERG GROUPS AND SHTERN'S QUESTION
}

\author{
MICHAEL MEGRELISHVILI
}

\begin{abstract}
Let $H(X):=(\mathbb{R} \times X) \lambda X^{*}$ be the generalized Heisenberg group induced by a normed space $X$. We prove that $X$ and $X^{*}$ are relatively minimal subgroups of $H(X)$. We show that the group $G:=H\left(L_{4}[0,1]\right)$ is reflexively representable but weakly continuous unitary representations of $G$ in Hilbert spaces do not separate points of $G$. This answers a question of A. Shtern [16].
\end{abstract}

\section{Groups of Heisenberg type}

Recall that the classical real 3-dimensional Heisenberg group can be defined as a linear group of the following matrices:

$$
\left(\begin{array}{lll}
1 & a & c \\
0 & 1 & b \\
0 & 0 & 1
\end{array}\right)
$$

where $a, b, c \in \mathbb{R}$. This group is isomorphic to the semidirect product $(\mathbb{R} \times \mathbb{R}) \lambda \mathbb{R}$ of $\mathbb{R} \times \mathbb{R}$ and $\mathbb{R}$.

We need a natural generalization (see, for example, [14, 12, 7]) which is based on biadditive mappings.

Definition 1.1. Let $E, F$ and $A$ be Hausdorff abelian topological groups and $w$ : $E \times F \rightarrow A$ be a continuous biadditive mapping. Denote by

$$
H(w)=(A \times E) 入 F
$$

the semidirect product (say, generalized Heisenberg group induced by $w$ ) of $F$ and the group $A \times E$. The resulting group, as a topological space, is the product $A \times E \times F$. The group operation is defined by the following rule: for a pair

$$
u_{1}=\left(a_{1}, x_{1}, f_{1}\right), \quad u_{2}=\left(a_{2}, x_{2}, f_{2}\right)
$$

define

$$
u_{1} \cdot u_{2}=\left(a_{1}+a_{2}+f_{1}\left(x_{2}\right), x_{1}+x_{2}, f_{1}+f_{2}\right)
$$

where, $f_{1}\left(x_{2}\right)=w\left(x_{2}, f_{1}\right)$.

Date: October 5, 2004.

Key words and phrases. Heisenberg group, unitary representation, minimal topological group, relatively minimal subgroup, weakly almost periodic, positive definite, reflexive space.

The author thanks to Israel Science Foundation (grant number 4699).

2000 Mathematical Subject Classification. 22A05, 43A60, 54H10. 
Then $H(w)$ becomes a two-step nilpotent Hausdorff topological group.

We will identify $E$ with $\left\{0_{A}\right\} \times E \times\left\{0_{F}\right\}$ and $F$ with $\left\{0_{A}\right\} \times\left\{0_{E}\right\} \times F$.

Elementary computations for the commutator $\left[u_{1}, u_{2}\right]$ give

$$
\left[u_{1}, u_{2}\right]=u_{1} u_{2} u_{1}^{-1} u_{2}^{-1}=\left(f_{1}\left(x_{2}\right)-f_{2}\left(x_{1}\right), 0_{E}, 0_{F}\right) .
$$

In the case of a normed space $X$ and the canonical bilinear function $w: X \times X^{*} \rightarrow \mathbb{R}$ we write $H(X)$ instead of $H(w)$. Clearly, the case of $H\left(\mathbb{R}^{n}\right)$ (induced by the inner product $w: \mathbb{R}^{n} \times \mathbb{R}^{n} \rightarrow \mathbb{R}$ ) gives the classical $2 \mathrm{n}+1$-dimensional Heisenberg group.

\section{Relatively Minimal SUbGROUPS}

First we need some definitions concerning the minimality concept.

Definition 2.1. Let $X$ be a subgroup of a Hausdorff topological group $(G, \tau)$. We say that $X$ is relatively minimal in $G$ if every coarser Hausdorff group topology $\sigma \subseteq \tau$ of $G$ induces on $X$ the original topology. That is, $\left.\sigma\right|_{X}=\left.\tau\right|_{X}$.

Equivalently, $X$ is relatively minimal in $G$ iff every injective continuous group homomorphism $G \rightarrow P$ into a Hausdorff topological group induces on $X$ a topological embedding. In particular, every faithful (that is, injective) weakly continuous unitary representation $G \rightarrow I s(H)$ for an arbitrary Hilbert space $H$ induces a topological group embedding (say, topologically faithful representation) of a relatively minimal subgroup $X$.

A minimal group in the sense of Stephenson [17] and Doïchinov [5] is just the group $G$ such that $G$ is relatively minimal in $G$. Recall some natural examples of minimal groups: the full unitary group $I s(H)$ (with the weak operator topology), the symmetric topological group $S_{X}, \mathbb{Z}$ with the $p$-adic topology, the semidirect product $\mathbb{R}^{n} \backslash \mathbb{R}_{+}$, every connected semisimple Lie group with finite center (e.g., $S L_{n}(\mathbb{R})$ ). Note that if $G$ is a locally compact abelian group with the canonical mapping $w: G \times G^{*} \rightarrow$ $\mathbb{T}$ then the corresponding generalized Heisenberg group $H(w)=(\mathbb{T} \times G) \lambda G^{*}$ is minimal [7, theorem 2.11]. Hence, every locally compact abelian group is a group retract of a minimal locally compact group. For more information see [3, 4, 6, 7, 8].

The following result seems to be new even for the classical 3-dimensional group $H(\mathbb{R})$.

Theorem 2.2. The subgroups $X$ and $X^{*}$ are relatively minimal in the generalized Heisenberg group $H(X)=(\mathbb{R} \times X) \lambda X^{*}$ for every normed space $X$.

Proof. Let $\tau$ be the given topology of $H(X)$ and suppose that $\sigma \subseteq \tau$ is a coarser Hausdorff group topology on $H(X)$. Denote by $X \times X^{*}$ the Banach space with respect to the norm $\|(x, f)\|:=\max \{\|x\|,\|f\|\}$. We prove in fact that the map

$$
q:(H(X), \sigma) \rightarrow X \times X^{*}, \quad(r, x, f) \mapsto(x, f)
$$

is continuous. This will imply that the natural retractions $(H(X), \sigma) \rightarrow X$ and $(H(X), \sigma) \rightarrow X^{*}$ are continuous. It guarantees the continuity of the identity maps $\left(X,\left.\sigma\right|_{X}\right) \rightarrow\left(X,\left.\tau\right|_{X}\right)$ and $\left(X^{*},\left.\sigma\right|_{X^{*}}\right) \rightarrow\left(X^{*},\left.\tau\right|_{X^{*}}\right)$. By the inclusion $\sigma \subseteq \tau$ we get $\left.\sigma\right|_{X}=\left.\tau\right|_{X}$ and $\left.\sigma\right|_{X^{*}}=\left.\tau\right|_{X^{*}}$. This will mean that $X$ and $X^{*}$ are relatively minimal subgroups in $H(X)$. 
Assuming the contrary there exists a coarser Hausdorff group topology $\sigma$ on $H(X)$ such that $q:(H(X), \sigma) \rightarrow X \times X^{*}$ is not continuous. Since $(H(X), \sigma)$ is a Hausdorff topological group, one can choose a $\sigma$-neighborhood $V$ of the neutral element $0:=$ $\left(0,0_{X}, 0_{X^{*}}\right)$ such that $1:=\left(1,0_{X}, 0_{X^{*}}\right) \neq[u, v]$ for every $u, v \in V$, where $[u, v]=$ $u v u^{-1} v^{-1}$.

Since the homomorphism $q$ is not $\sigma$-continuous (at $\mathbf{0}$ ) there exists a positive $\delta$ such that $q(U)$ is not embedded into the ball

$$
B\left(\left(0_{X}, 0_{X^{*}}\right), \delta\right):=\left\{(x, f) \in X \times X^{*}: \max \{\|x\|,\|f\|\}<\delta\right\}
$$

for every $\sigma$-neighborhood $U$ of $\mathbf{0}$. Then (similar to [7, Lemma 3.5]) it follows that $q(U)$ is norm-unbounded in $X \times X^{*}$. Indeed, for every $n \in \mathbb{N}$ choose a $\sigma$-neighborhood $W$ of $\mathbf{0}$ such that

$$
\underbrace{W \cdot W \cdots W}_{n} \subseteq U
$$

As we already know, $q(W)$ is not a subset of $B\left(\left(0_{X}, 0_{X^{*}}\right), \delta\right)$. Therefore for every $n \in \mathbb{N}$ there exists a triple $t_{n}:=\left(r_{n}, x_{n}, f_{n}\right) \in W$ such that the pair $\left(x_{n}, f_{n}\right)=q\left(t_{n}\right)$ satisfies $\left\|\left(x_{n}, f_{n}\right)\right\|:=\max \left\{\left\|x_{n}\right\|,\left\|f_{n}\right\|\right\} \geq \delta$ in $X \times X^{*}$. Then by the definition of the group operation in $H(X)$ we get $t_{n}^{n}=\left(s_{n}, n x_{n}, n f_{n}\right)$ (for some $s_{n} \in \mathbb{R}$ ). Thus, $\left\|q\left(t_{n}^{n}\right)\right\|=\left\|\left(n x_{n}, n f_{n}\right)\right\| \geq n \delta$. Therefore, $q(U)$ is norm-unbounded. Then there exists a sequence $S:=\left\{u_{n}:=\left(a_{n}, y_{n}, \phi_{n}\right)\right\}_{n \in \mathbb{N}}$ in $U$ such that at least one of the sets $A:=\left\{y_{n}\right\}_{n \in \mathbb{N}}$ and $B:=\left\{\phi_{n}\right\}_{n \in \mathbb{N}}$ is unbounded. Suppose first that $A$ is unbounded. The intersection

$$
V_{X^{*}}:=V \cap X^{*}
$$

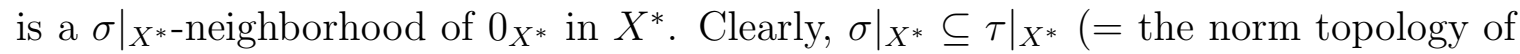
$\left.X^{*}\right)$. Therefore, $V_{X^{*}}$ contains a ball $B\left(0_{X^{*}}, \varepsilon_{0}\right)$ of $X^{*}$ for some $\varepsilon_{0}>0$. Since $A$ is norm-unbounded and $B\left(0_{X^{*}}, \varepsilon_{0}\right) \subseteq V_{X^{*}}$, Hahn-Banach theorem implies that the set

$$
<A, V_{X^{*}}>=\left\{<y_{n}, f>=f\left(y_{n}\right): \quad n \in \mathbb{N}, f \in V_{X^{*}}\right\}
$$

is unbounded in $\mathbb{R}$. In fact we have $\left\langle A, V_{X^{*}}>=\mathbb{R}\right.$ because $<,>: X \times X^{*} \rightarrow \mathbb{R}$ is bilinear and $c B\left(0_{X^{*}}, \varepsilon_{0}\right) \subseteq B\left(0_{X^{*}}, \varepsilon_{0}\right)$ for every $c \in[-1,1]$.

On the other hand, for every $u_{n}=\left(a_{n}, y_{n}, \phi_{n}\right) \in S \subset V$ and $f=\left(0,0_{X}, f\right) \in$ $V_{X^{*}} \subset V$, the corresponding commutator $\left[f, u_{n}\right]$ is $\left(f\left(y_{n}\right), 0_{X}, 0_{X^{*}}\right)$. Hence, $[V, V]=$ $\{[a, b]: a, b \in V\}$ contains the subgroup $\mathbb{R} \times\left\{0_{X}\right\} \times\left\{0_{X^{*}}\right\}$. But then $\mathbf{1} \in[V, V]$. This contradicts our assumption.

The case of unbounded $B=\left\{\phi_{n}\right\}_{n \in \mathbb{N}}$ is similar. Indeed, observe that we have

$$
<V, V>\supseteq<V_{X}, B>\supseteq<B\left(0_{X}, \varepsilon_{0}\right), B>=\mathbb{R}
$$

for every $B\left(0_{X}, \varepsilon_{0}\right) \subseteq V_{X}:=V \cap X$. On the other hand, $\left[u_{n}, x\right]=\left(\phi_{n}(x), 0_{X}, 0_{X^{*}}\right)$ for every $u_{n} \in S$ and every $x:=\left(0, x, 0_{X^{*}}\right) \in V_{X}$. As before, this implies that $\mathbf{1} \in[V, V]$.

Note that the subgroups $X$ and $X^{*}$ being relatively minimal in $H(X)$ are not however minimal because every abelian minimal group necessarily is precompact (see for example [3] ). 


\section{An Applichtion: Shtern's Question}

Let $I s(X)$ be the group of all linear isometries of a Banach space $X$. Note that by [10] the strong and weak operator topologies on $I s(X)$ coincide for every reflexive $X$. For some related recent results about infinite-dimensional representations of general topological groups we refer to [13].

As an application of our results we prove the following theorem which answers a question of A. Shtern [16] (for a weaker version see [11, Theorem 3.1] which states that the additive topological group $L_{4}[0,1]$ is not embedded into $I s(H)$ for any Hilbert space $H$ ).

Theorem 3.1. There exists a topological group $G$ such that:

(a) Weakly continuous unitary representations of $G$ in Hilbert spaces do not separate points of $G$.

(b) $G$ is a topological subgroup of $I s(X)$ for some reflexive Banach space $X$, where $I s(X)$ is endowed with the strong (equivalently, weak) operator topology.

We claim that the desired group $G$ is the Heisenberg group of the canonical bilinear mapping

That is,

$$
w: L_{4}[0,1] \times L_{\frac{4}{3}}[0,1] \rightarrow \mathbb{R}
$$

$$
G:=H\left(L_{4}\right)=\left(\mathbb{R} \times L_{4}\right) 入 L_{\frac{4}{3}} .
$$

First we prove assertion (a) of Theorem 3.1 .

Lemma 3.2. Weakly continuous unitary representations of the generalized Heisenberg group $G:=H\left(L_{4}\right)$ in Hilbert spaces do not separate points.

Proof. Assuming the contrary holds true, let $\mathcal{F}=\left\{h_{i}: G \rightarrow I s\left(H_{i}\right): i \in I\right\}$ be some point separating set of weakly continuous unitary representations of $G$. Consider the corresponding $l_{2}$-sum $H:=\bigoplus_{i \in I} H_{i}$ of Hilbert spaces. Passing to the naturally defined $l_{2}$-sum of representations we get a weakly continuous representation $h: G \rightarrow$ $I s(H)$ which is faithful because $\mathcal{F}$ separates the points. By the relative minimality of the subgroup $L_{4}$ in $G$ (Theorem 2.2), we must conclude that the restriction map $\left.h\right|_{L_{4}}$ : $L_{4} \rightarrow I s(H)$ necessarily is a topological group embedding. Therefore, there exists a topologically faithful unitary representation of $L_{4}$ into the unitary group $\left.I s(H)\right)$. This contradicts [11, Theorem 3.1].

We say that a map $F: A \times B \rightarrow \mathbb{R}$ has the Double Limit Property (in short: DLP) if for every pair of sequences $\left\{a_{n}\right\}_{n \in \mathbb{N}},\left\{b_{m}\right\}_{m \in \mathbb{N}}$ in $A$ and $B$ respectively,

$$
\lim _{m} \lim _{n} F\left(a_{n}, b_{m}\right)=\lim _{n} \lim _{m} F\left(a_{n}, b_{m}\right)
$$

holds whenever both of the limits exist. Let us say that a continuous function $\phi$ : $G \rightarrow \mathbb{R}$ on a topological group $G$ has the DLP if the induced map

$$
F: G \times G \rightarrow \mathbb{R}, F(x, y):=\phi(x y)
$$

has the DLP.

We collect here some auxiliary facts. 
Lemma 3.3. (1) (Grothendieck's characterization of weakly almost periodicity; see for example, [1]) A bounded continuous function $\phi: G \rightarrow \mathbb{R}$ is weakly almost periodic (wap, for short) iff $\phi$ has the DLP.

(2) (16] and [11, Fact 2.1]) A topological group $G$ can be embedded into Is $(X)$ (endowed with the strong (equivalently, weak) operator topology) for some reflexive Banach space $X$ iff the algebra $W A P(G)$ of all wap functions separates the neutral element from closed subsets of $G$.

(3) Let $\left\{a_{n, m}\right\}_{n, m \in \mathbb{N}}$ be a bounded double sequence of real numbers. Then there exists a double subsequence $\left\{a_{i_{n}, j_{m}}\right\}_{n, m \in \mathbb{N}}$ such that the double limits $c_{1}:=$ $\lim _{m} \lim _{n} a_{i_{n}, j_{m}}$ and $c_{2}:=\lim _{n} \lim _{m} a_{i_{n}, j_{m}}$ both exist.

(4) Let $X$ be a reflexive Banach space with the canonical bilinear mapping

$$
X \times X^{*} \rightarrow \mathbb{R}, \quad(x, f) \mapsto f(x) .
$$

Then for every pair of bounded subsets $A \subset X, F \subset X^{*}$ the restriction map $A \times F \rightarrow \mathbb{R}$ has the DLP.

(5) 11, Lemma 3.4] The norm in the Banach space $L_{2 k}([0,1])$ has the DLP for every $k \in \mathbb{N}$.

Proof. (3): There exists a real number $r$ such that $a_{n, m} \in[-r, r]$ for every $n, m \in \mathbb{N}$. Consider the sequence $\left\{v_{n}\right\}_{n \in \mathbb{N}}$, where $v_{n}:=\left(a_{n, 1}, a_{n, 2}, \cdots\right)$. Every $v_{n}$ can be treated as an element of the compact metrizable space $[-r, r]^{\mathbb{N}}$. We can choose a subsequence $\left\{v_{i_{n}}\right\}_{n \in \mathbb{N}}$ which converges to some $v:=\left(t_{1}, t_{2}, \cdots\right) \in[-r, r]^{\mathbb{N}}$. Then $\lim _{n} a_{i_{n}, m}=t_{m}$ holds for every $m \in \mathbb{N}$. Since $\left\{t_{m}\right\}_{m \in N}$ is bounded we can find a sequence $j_{1}<$ $j_{2}<\cdots$ and a real number $c_{1} \in[-r, r]$ such that $\lim _{m} t_{j_{m}}=c_{1}$. It follows that $c_{1}=\lim _{m} \lim _{n} a_{i_{n}, j_{m}}$. Now starting with the double sequence $\left\{a_{i_{n}, j_{m}}\right\}_{n, m \in \mathbb{N}}$ we obtain by similar arguments (switching the roles of $n$ and $m$ ) its double subsequence such that the second double limit $c_{2}$ also exists.

(4): Let $f_{n}$ and $a_{m}$ be two sequences respectively in $F$ and $A$ such that $c_{1}=$ $\lim _{m} \lim _{n} f_{n}\left(a_{m}\right)$ and $c_{2}=\lim _{n} \lim _{m} f_{n}\left(a_{m}\right)$. By the reflexivity, the subsets $F$ and $A$ are relatively weakly compact in $X^{*}$ and $X$ respectively. Eberlein-Šmulian theorem implies that $F$ and $A$ are relatively sequentially compact with respect to the weak topologies. Hence there exist a subsequence $f_{i_{n}}$ of $f_{n}$ and a subsequence $a_{j_{m}}$ of $a_{m}$ such that the weak $\operatorname{limits} \lim _{n} f_{i_{n}}=f$ and $\lim _{m} a_{j_{m}}=a$ are defined in $X^{*}$ and $X$. Since the map $(X$, weak $) \times\left(X^{*}\right.$, weak $\left.k^{*}\right) \rightarrow \mathbb{R}$ is separately continuous we obtain

$$
\begin{aligned}
& c_{1}=\lim _{m} \lim _{n} f_{n}\left(a_{m}\right)=\lim _{m} \lim _{n} f_{i_{n}}\left(a_{j_{m}}\right)=\lim _{m} f\left(a_{j_{m}}\right)=f(a) \\
& c_{2}=\lim _{n} \lim _{m} f_{n}\left(a_{m}\right)=\lim _{n} \lim _{m} f_{i_{n}}\left(a_{j_{m}}\right)=\lim _{n} f_{i_{n}}(a)=f(a) .
\end{aligned}
$$

Thus, $c_{1}=c_{2}$, as desired.

The norm of the Banach space $c_{0}$ does not satisfy the DLP. Indeed, define $u_{n}:=e_{n}$ (the standard basis vectors) and $v_{m}:=\sum_{i=1}^{m} e_{i}$. Then

$$
1=\lim _{m} \lim _{n}\left\|u_{n}+v_{m}\right\| \neq \lim _{n} \lim _{m}\left\|u_{n}+v_{m}\right\|=2 .
$$

Note also that by [9] there exists a non-trivial Hausdorff topological group $G$ (namely, the group $G:=H_{+}[0,1]$ of all orientation preserving selfhomeomorphisms of the closed interval $[0,1]$ ) such that every (weakly) continuous representation $h$ : 
$G \rightarrow I s(X)$ is constant for every reflexive $X$. Equivalently, every wap function on $G$ is constant.

Now we prove assertion (b) of Theorem 3.1 .

Lemma 3.4. The generalized Heisenberg group $G:=H\left(L_{4}\right)$ is reflexively representable. That is, there exists a reflexive Banach space $X$ such that $G$ is a topological subgroup of Is $(X)$ endowed with the strong (equivalently, weak) operator topology.

Proof. Define the following continuous bounded real valued function

$$
\phi: G=\left(\mathbb{R} \times L_{4}\right) 入 L_{\frac{4}{3}} \rightarrow \mathbb{R}, \quad \phi(r, x, f)=\frac{1}{1+|r|+\|x\|+\|f\|}
$$

This function separates the neutral element $\mathbf{0}=(0,0,0)$ from closed subsets $\mathbf{0} \notin A$ in $G$. So, by Lemma 3.32 , it suffices to establish that $\phi$ is wap. Assuming the contrary holds true we get by Lemma 3.31 that $\phi$ does not satisfy the DLP. Therefore there exist two sequences $u_{n}=\left(a_{n}, x_{n}, p_{n}\right), v_{m}=\left(b_{m}, y_{m}, q_{m}\right)$ in $G$ such that for the double sequence

$$
\phi\left(u_{n} v_{m}\right)=\frac{1}{1+\left|a_{n}+b_{m}+p_{n}\left(y_{m}\right)\right|+\left\|x_{n}+y_{m}\right\|+\left\|p_{n}+q_{m}\right\|} .
$$

the double limits

$$
s_{1}:=\lim _{m} \lim _{n} \phi\left(u_{n} v_{m}\right), \quad s_{2}:=\lim _{n} \lim _{m} \phi\left(u_{n} v_{m}\right)
$$

exist but $s_{1} \neq s_{2}$.

We can assume without restricting of generality (up to the subsequences) that all sequences

$$
a_{n}, x_{n}, p_{n}, b_{m}, y_{m}, q_{m}
$$

are bounded. Indeed, if one of the sequences above is unbounded, passing to appropriate subsequences, we get that the corresponding double limits both are 0. Actually we can and do assume that the sequences $a_{n}$ and $b_{m}$ converge in $\mathbb{R}$. Moreover, by Lemma 3.3 .3 we can suppose in addition that each of the following three (bounded) real valued double sequences

$$
\left\|p_{n}+q_{m}\right\|, \quad\left\|x_{n}+y_{m}\right\|, \quad p_{n}\left(y_{m}\right)
$$

have double limits. Now it suffices to show that in each one of the cases (a), (b), and (c) below the corresponding double limits are the same. This will imply that $s_{1}=s_{2}$, providing the desired contradiction.

(a) First we check that $\lim _{m} \lim _{n}\left\|p_{n}+q_{m}\right\|=\lim _{n} \lim _{m}\left\|p_{n}+q_{m}\right\|$. We have to show that the function $L_{\frac{4}{3}} \rightarrow \mathbb{R}, f \mapsto\|f\|$ has the DLP. It suffices to establish that the function $f \mapsto e^{-\|f\|^{p}}$ has the DLP on $L_{\frac{4}{3}}$. But this function is positive definite on $L_{p}$ for every $1 \leq p \leq 2$ by a classical result of Shoenberg [15]. On the other hand every continuous positive definite function on a topological group is wap (see [2, Corollary 3.3]).

(b) Observe also that the map $L_{4} \rightarrow \mathbb{R}, x \mapsto\|x\|$ has the DLP by Lemma 3.3.5. Therefore, $\lim _{m} \lim _{n}\left\|x_{n}+y_{m}\right\|=\lim _{n} \lim _{m}\left\|x_{n}+y_{m}\right\|$ holds. 
(c) Finally we show that $\lim _{m} \lim _{n} p_{n}\left(y_{m}\right)=\lim _{n} \lim _{m} p_{n}\left(y_{m}\right)$. Note that, as before, by our assumptions, the sequences $p_{n}$ and $y_{m}$ are bounded in $L_{\frac{4}{3}}$ and $L_{4}$ respectively and the $\operatorname{limits} c_{1}:=\lim _{m} \lim _{n} p_{n}\left(y_{m}\right), c_{2}:=\lim _{n} \lim _{m} p_{n}\left(y_{m}\right)$ both exist. Now the equality $c_{1}=c_{2}$ directly follows by Lemma [3.3, 4 .

The proof of Theorem 3.1 follows now from Lemmas 3.2 and 3.4 ,

Finally we thank D. Dikranjan, N. Krupnik and A. Shtern for useful suggestions.

\section{REFERENCES}

1. J.F. Berglund, H.D. Junghenn, P. Milnes, Analysis on semigroups, Canad. Math. Soc., WileyInterscience Publication, 1989.

2. R.B. Burckel, Weakly almost periodic functions on semigroups, Gordon and Breach Science Publishers, New York-London-Paris, 1970.

3. D. Dikranjan, Iv. Prodanov, L. Stoyanov, Topological groups: characters, dualities and minimal group topologies, Pure and Appl. Math. 130, Marcel Dekker, New York-Basel, 1989.

4. D. Dikranjan, Recent progress in minimal topological groups, Topology Applications, 85 (1998), 53-91.

5. D. Doïtchinov, Produits de groupes topologiques minimaux, Bull. Sci. Math. (2) 97 (1972) 59-64.

6. D. Gamarnik, Minimality of the group Aut(C), Serdika 17:4, (1991), 197-201.

7. M. Megrelishvili, Group representations and construction of minimal topological groups, Topology Applications, 62 (1995), 1-19.

8. M. Megrelishvili, G-Minimal Topological Groups, In: Abelian Groups, Module Theory and Topology, Lecture Notes in Pure and Applied Algebra, Marcel Dekker, 201 (1998), 289-300.

9. M. Megrelishvili, Every semitopological semigroup compactification of the group $H_{+}[0,1]$ is trivial, Semigroup forum 63, (2001), 357-370.

10. M. Megrelishvili, Operator topologies and reflexive representability, in: "Nuclear groups and Lie groups", Research \& Exposition in Mathematics, Heldermann, Verlag-Berlin, 24 (2001), 189-201.

11. M. Megrelishvili, Reflexively but not unitarily representable topological groups, Topology Proceedings, 25, 2002, 615-625.

12. P. Milnes, Enveloping semigroups, distal flows and groups of Heisenberg type, Houston Journal of Mathematics, 15:4 (1989), 563-572.

13. V.G. Pestov, Topological groups: where to from here?, Topology Proceedings, 24 (1999), 421502. http://arXiv.org/abs/math.GN/9910144.

14. H. Reiter, Uber den Satz von Wiener and lokalkompakte Gruppen, Comm. Math. Helv., 49 (1974), 333-364.

15. I.J. Shoenberg, Metric spaces and positive definite functions, Trans. AMS, 44 (1938), 522-536.

16. A. Shtern, Compact semitopological semigroups and reflexive representability of topological groups, Russ. J. Math. Physics 2 (1994), 131-132.

17. R. Stephenson, Minimal topological groups, Math. Ann., 192 (1971), 193-195.

Department of Mathematics, Bar-Ilan University, 52900 Ramat-Gan, Israel

E-mail address: megereli@math.biu.ac.il

$U R L:$ http://www.math.biu.ac.il/ megereli 